\title{
Worldwide Distribution of Moyamoya Disease
}

\author{
Yasunobu GoTo and Yasuhiro YoNEKAWA
}

Department of Neurosurgery, National Cardiovascular Center, Suita, Osaka

\begin{abstract}
Many cases of spontaneous occlusion of the circle of Willis (moyamoya disease) have been reported in Japan, but the worldwide distribution is unclear. The total number of cases in each country reported in the literature and complications were evaluated. A total of 1063 cases have occurred worldwide excluding Japan. Cases were observed most frequently in Asia and in people of non-Caucasian origin.
\end{abstract}

Key words: moyamoya disease, epidemiology

\section{Introduction}

Since moyamoya disease was first described in $1955,^{8)}$ many cases have occurred worldwide. A nationwide survey in 1989 estimated the number of patients in Japan to be about $3000 .^{7)}$ However, the prevalence in other countries remains unclear. Therefore, we reviewed the past 18 years of world literature to ascer- tain the distribution, specificity, and complications of this curious disease.

\section{Materials and Methods}

Literature in English from Index Medicus between 1972 and 1989 was surveyed. All papers were classified by running titles relating to this disease:

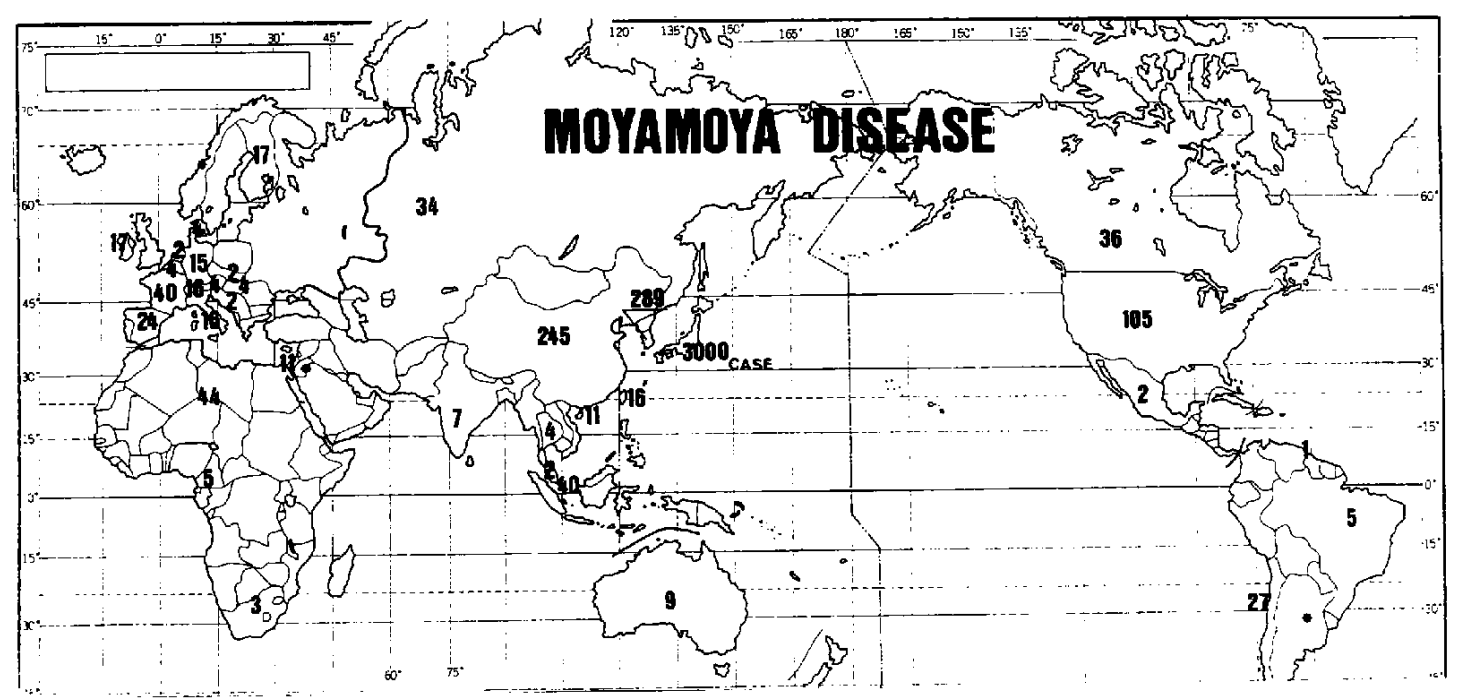

Fig. 1 Worldwide distribution of moyamoya disease. Number indicates total moyamoya disease cases in literature per country. Asterisk shows reports from this country but the number of cases is unknown.

Received August 7, 1991; Accepted April 13, 1992

Author's present address: Y. Goto, M.D., Department of Neurosurgery, University of Virginia Health Sciences Center, Charlottesville, Virginia, U.S.A. 
moyamoya disease, moyamoya syndrome, moyamoya vessels, rete mirabile, and spontaneous occlusion of the circle of Willis. The number of cases, complications, the institution of the authors, and the country were recorded. The total number in each country was calculated by summing the number of cases in the literature. Japanese papers were excluded because the annual occurrence and prevalence are already available.

\section{Results}

There were 1063 cases of moyamoya disease reported worldwide, excluding Japan (Fig. 1, Table 1); 625 occurred in Asia, including 245 in China and 289 in Korea, ${ }^{2)} 201$ in Europe, 176 in North and South America, 52 in Africa, and nine in Oceania.

Table 2 shows the complications associated with the disease: Inflammatory diseases, leptospirosis and meningitis (e.g. tuberculous meningitis) occurred frequently or were suggested as the cause. Blood diseases such as sickle cell anemia and Fanconi's anemia were also frequent. Other observations included genetic disorders such as phacomatosis and Down's syndrome, and radiation-induced disorders.

\section{Discussion}

A survey conducted by Kudo ${ }^{6}$ showed no regional clustering of moyamoya disease in Japan. The disease has only been reported sporadically worldwide. Yonekawa et al. ${ }^{11)}$ showed that it does occur throughout the world. Kitamura et al. ${ }^{4,5)}$ reported 568 cases of moyamoya disease from data collected mainly from South-East Asia. Our review of the literature also found cases throughout the world, but particularly in patients of Asiatic origin. However, the etiological factors of this disease have not yet been clarified.

The number of cases reported in each region does not directly reflect the incidence because of considerable differences in density of population, level of medical care, especially the availability of cerebral angiography, and the awareness of this disease. Interestingly, many cases in North America, South America, and Europe were Asians, immigrants from Asia, or Indians. Some patients in France and England were non-Caucasian, many of them being Africans. These findings suggest an association with ethnotype, with a lower reported incidence in Caucasians.

The etiology of moyamoya disease is unclear. In China, the association of moyamoya disease with leptospirosis ${ }^{1)}$ has attracted attention. In Japan, the in-
Table 1 Reported cases of moyamoya disease in the literature*

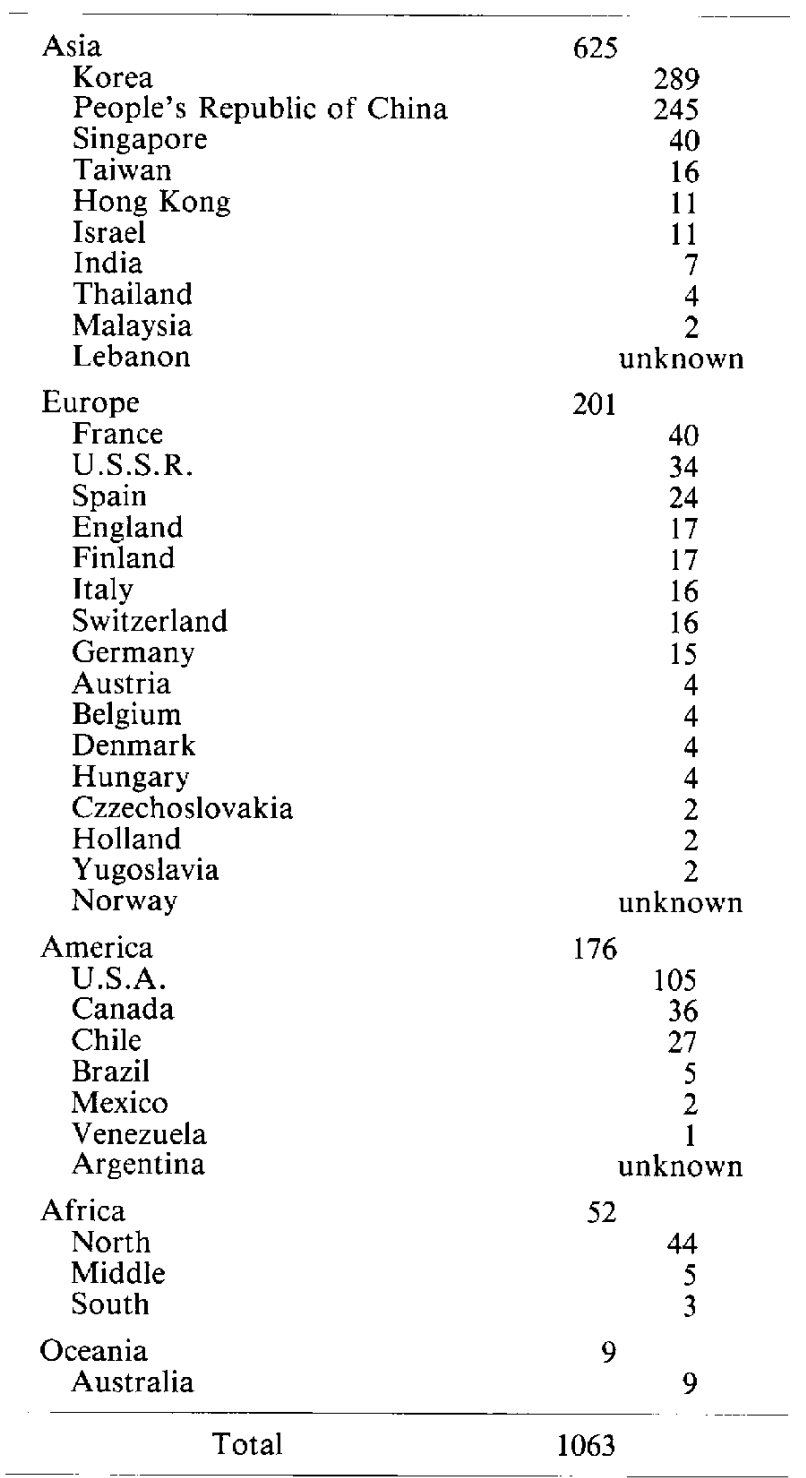

${ }^{*}$ Between 1972 and 1989, Japan excluded.

volvement of abnormal sympathetic function associated with chronic respiratory tract infection (tonsillitis and pharyngolaryngitis) has been suggested. Yamada et $a l^{10)}$ reported that the antibody titers against Propionibacterium acnes were significantly increased in patients with this disease. Studies on autoantibody ${ }^{9)}$ or human leukocyte antigen have shown no clear causative factors. Kawai and Fukuyama ${ }^{3}$ analyzed a large number of families and reported that familial clustering and multifactorial inheritance were strongly suggested.

The complications identified by our review included tumors and radiation-induced changes resem- 
Table 2 Disorders associated with moyamoya disease

\author{
Infectious disease \\ leptospirosis \\ meningitis (tuberculosis, etc.) \\ arteritis \\ nephritis \\ tonsillitis \\ Hematological disorders \\ sickle cell anemia \\ Fanconi's anemia \\ thalassemia \\ Metabolic disorders \\ hyperlipoproteinemia (type $2 \mathrm{~A}$ ) \\ glycogen storage disease (type 1) \\ lipohyalinosis \\ pseudoxanthoma elasticum \\ hyperthyroidism \\ impaired NADH-COQ reductase activity \\ Genetic, chromosomal disorders \\ von Recklinghausen's disease \\ tuberous sclerosis \\ Down's syndrome (21-trisomy) \\ Turner's syndrome \\ Congenital anomalies \\ clover leaf skull \\ transposition of the great arteries \\ Neoplasms \\ parasellar tumor (craniopharyngioma, etc.) \\ Wilms' tumor \\ Drug use \\ phenobarbital \\ oral contraceptives \\ Others \\ cardiomyopathy \\ autoimmune disease \\ renal artery stenosis \\ fibromuscular dysplasia \\ cranial trauma \\ cranial irradiation \\ arteriovenous malformation \\ cerebral aneurysm \\ identical twins \\ harlequinism \\ growth failure \\ Alagille syndrome \\ polycystic kidney and eosinophilic granuloma
}

NADH-CoQ: dihydronicotinamide adenine dinucleotide-coenzyme Q.

bling moyamoya disease (moyamoya syndrome). Whether the morphological abnormality of erythrocytes and previous use of drugs are associated with the pathogenesis of this disease or are coincidental is unclear.

The association of moyamoya disease with vascular lesions such as arteriovenous malformation, transposition of great arteries, renal artery stenosis, or fibromuscular dysplasia, coincidental cerebral aneurysms, and peripheral pseudoaneurysms caused by hemodynamic stress specific to moyamoya disease have been reported, but may or may not be coincidental. According to the diagnostic guidelines for moyamoya disease established by the Research Committee on Spontaneous Occlusion of the Circle of Willis sponsored by the Ministry of Health and Welfare, Japan (Table 3), cases showing apparent underlying diseases are excluded. In this respect, our review did not strictly differentiate moyamoya syndrome and moyamoya disease. However, complications to be included or excluded have not yet been determined. Therefore, this study recorded all associated disorders.

We conclude that the diagnostic criteria require further evaluation and distribution worldwide to clarify the distribution of moyamoya disease and its pathogenesis.

\section{Acknowledgments}

This work has been supported by the Research Committee on Spontaneous Occlusion of the Circle of Willis sponsored by the Ministry of Health and Welfare, Japan.

\section{References}

1) Cheng MK: A review of cerebrovascular surgery in People's Republic of China. Stroke 13: 249-255, 1982

2) Choi KS: Moyamoya disease in Korea. A cooperative study, in Suzuki J (ed): Advances in Surgery for Cerebral Stroke. Tokyo, Springer-Verlag, 1988, pp 107-109

3) Kawai M, Fukuyama $Y$ : A genetic study of idiopathic spontaneous multiple occlusions of the circle of Willis (moyamoya disease). Tokyo Joshi Ika Daigaku Zasshi 55: 427-441, 1985 (in Japanese)

4) Kitamura $\mathrm{K}$ : Introduction of round table discussion on moyamoya disease at the International Symposium on Surgery for Cerebral Stroke, in: Annual Report 1987 of the Research Committee on Spontaneous Occlusion of the Circle of Willis. 1988, pp 19-21 (in Japanese)

5) Kitamura K, Fukui M, Oka K, Matsushima $T$, Kurokawa T, Hasuo K: Moyamoya disease, in Tool JF (ed): Handbook of Clinical Neurology, vol 11 . Amsterdam, Elsevier Science Publishers, 1989, pp 293-306

6) Kudo T: General aspects, in Kudo T (ed): A Disease with Abnormal Intracranial Vascular Networks. Spontaneous Occlusion of the Circle of Willis. Tokyo, Igaku Shoin, 1967, p 1 (in Japanese)

7) Sasaki R, Suzuki S, Tamakoshi A, Oono $Y$, Yanagawa $Y$, Murase T, Mutou Y, Moriwaki $H$, Onchi $\mathrm{M}$ : Prevalence of intractable diseases by 
Table 3 Guidelines for the diagnosis of moyamoya disease*

1. i-a) Age of onset varies but is prevalent in youth and in females. Familial occurrence is sometimes reported.

i-b) Symptoms and types of progression vary, with an asymptomatic type and a type with transient or persistent neurological deficits of slight or severe degree.

i-c) Cerebral ischemia is observed, usually in juvenile patients and hemorrhage in adult patients.

ii) In juveniles, hemiparesis, monoparesis, sensory disturbance, involuntary movement, headache, and convulsion recur, sometimes on alternating sides. Mental retardation or persistent neurological deficits are also observed. The hemorrhagic type is rare.

iii) In adults, symptoms similar to those observed in juveniles may appear, but most have a sudden onset of intraventricular, subarachnoid, or intracerebral hemorrhage. Patients usually recover from the bleeding with or without persistent deficits, but deaths have also been noted in severe cases.

2. Angiography is indispensable for the diagnosis. The following findings are observed.

i) Stenosis or occlusion is observed at the terminal portion of the intracranial ICA and/or at the proximal portion of the ACA and/or the MCA.

ii) Abnormal vascular moyamoya networks are observed in the vicinity of the above mentioned areas in the arterial phase.

iii) These findings are recognized bilaterally.

3. Etiology is unknown, and an underlying disease (such as atherosclerosis, meningitis, Down's syndrome, neoplasms, von Recklinghausen's disease, post-irradiation) is ruled out.

4. Pathological findings helpful for the diagnosis:

i) Intimal thickening and resulting stenosis or occlusion are observed around the intracranial terminal portion of the ICA usually bilaterally. They are sometimes associated with lipid degeneration.

ii) In the main arteries (ACA, MCA, PcomA) comprising the circle of Willis, various degrees of stenosis and occlusion are observed in association with intimal fibrous thickening, and widening of the internal elastic lamina and tunica media.

iii) Many small vascular channels (perforators and anastomotic branches) are observed around the circle of willis.

iv) Small networks of vessels are observed in the pia mater.

*Established by Research Committee on Spontaneous Occlusion of the Circle of Willis, Ministry of Health and Welfare, Japan, 1988. Diagnostic criteria are divided into two groups, as follows, with reference to 1 above. Autopsy cases in which angiography was not performed will be investigated separately with reference to 4 . Definite case: fulfills all findings described in 2 and 3 simultaneously. Juvenile cases fulfill 2-i) and ii) on one side and remarkable stenosis at the terminal portion of the intracranial ICA on the other side. Probable case: fulfills all criteria for definite cases except 2-iii). ACA: anterior cerebral artery, ICA: internal carotid artery, MCA: middle cerebral artery, PcomA: posterior communicating artery.

nationwide survey, in: Annual Report 1990 of Epidemiology of the Intractable Diseases Research Committee. 1991, pp 27-29 (in Japanese)

8) Takeuchi K, Shimizu K: Hypoplasia of the bilateral internal carotid arteries. Brain and Nerve (Tokyo) 9: 37-43, 1957 (in Japanese)

9) Wanifuchi $H$, Kagawa $M$, Takeshita $M$, Izawa $M$, Maruyama S, Kitamura K: Autoimmune antibody in moyamoya disease. No Shinkei Geka 14: 31-35, 1986 (in Japanese)

10) Yamada H, Deguchi K, Sakai N, Ando T, Ueno K, Watanabe K: Clinical analysis of serum antibody to Propionibacterium acnes and serum protein in moyamoya disease, in: Annual Report 1988 of the Research Committee on Spontaneous Occlusion of the Circle of Willis. 1989, pp 69-73 (in Japanese)

11) Yonekawa $Y$, Handa $H$, Okuno $T$ : Moyamoya disease: Diagnosis, treatment and recent achievement, in Barnett HJM (ed): Stroke, vol 2. New York, Churchill Livingstone, 1986, pp 805-829

Address reprint requests to: Y. Yonekawa, M.D., Department of Neurosurgery, National Cardiovascular Center, 5-7-1 Fujishirodai, Suita, Osaka 565, Japan. 